\title{
Multi-mode Fiber Coarse WDM Grating Router Using Broadband Add/Drop Filters for Wavelength Re-Use
}

\author{
R.R. Patel \\ S.W. Bond \\ M.C. Larson \\ M.D. Pocha \\ H.E. Garrett \\ M.E. Lowry \\ R.J. Deri
}

This paper was prepared for submittal to the

Institute of Elcctrical and Electronics Engineers

Lasers and Electro-Optics Society Annual Meeting 1999

San Francisco, CA

November 8-11, 1999

June 1999

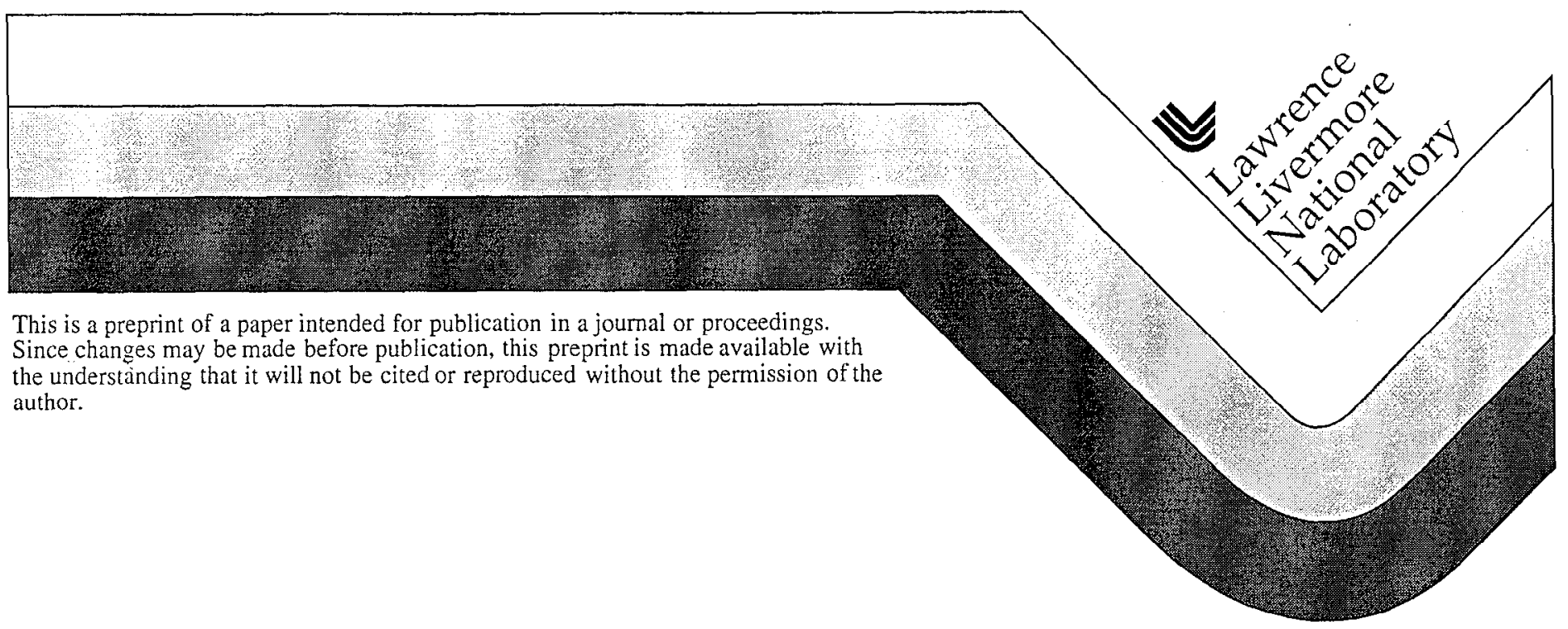




\section{DISCLAIMER}

This document was prepared as an account of work sponsored by an agency of the United States Government. Neither the United States Government nor the University of California nor any of their employees, makes any warranty, express or implied, or assumes any legal liability or responsibility for the accuracy, completeness, or usefulness of any information, apparatus, product, or process

disclosed, or represents that its use would not infringe privately owned rights. Reference herein to any specific commercial product, process, or service by trade name, trademark, manufacturer, or otherwise, does not necessarily constitute or imply its endorsement, recommendation, or favoring by the United States Government or the University of California. The views and opinions of authors expressed herein do not necessarily state or reflect those of the United States Government or the University of California, and shall not be used for advertising or product endorsement purposes. 


\title{
Multi-mode Fiber Coarse WDM Grating Router Using Broadband Add/Drop Filters for Wavelength Re-Use
}

\author{
R. R. Patel, S. W. Bond, M. C. Larson, M. D. Pocha, H. E. Garrett, M. E. Lowry, and R. J. \\ Deri*
}

Lawrence Livermore National Laboratory

L-174 Livermore, CA 94550, email:pate16@llnl.gov

*R J. Deri is with Terawave Communications Inc., 30695 Huntwood Avenue Hayward, CA 94544

We demonstrate a grating-router with 37nm channel spacing and 6nm FWHM in the 800-900nm range for WDM over multimode fiber. Broadband thin-film add/drop filters provide wavelength re-use enabling NxN fully nonblocking interconnection with $\mathrm{N}$ wavelengths.

\section{Introduction}

For single mode fiber (SMF) applications the arrayed waveguide grating router (AWG) provides passive wavelength routing with spectral channels being used more than once in the routing table to achieve full $\mathrm{NxN}$ interconnection with only $\mathrm{N}$ wavelengths[1]. AWGs cannot be used with MMF due to the excessive losses in coupling from MMF to single mode waveguides. We report the development of a wavelength router ( $\mathrm{NxN}$ wavelength multiplexer) for use in MMF based optical networks. The device uses a blazed diffraction grating and broadband add/drop filters to provide wavelength re-use thus enabling fully non-blocking $\mathrm{NxN}$ interconnection with only $N$ wavelengths. Initial cxperimental results using 3 inputs and 3 outputs arc presented.

\section{Device Configuration}

Figure 1 shows the experimental setup. In this figure, the inputs $\mathrm{A}, \mathrm{B}$, and $\mathrm{C}$ are mapped to outputs $1,2,3$, $1 *$, and $2^{*}$, which are subsequently combined with add/drop filters to produce the final 3 outputs $\mathrm{X}, \mathrm{Y}$, and $\mathrm{Z}$. Wavelength routing was demonstrated using 3 wavelength channels: 827, 864, and $900 \mathrm{~nm}$. Graded index (GRIN) $62.5 / 125 \mu \mathrm{m}$ MMF inputs and outputs were terminated in an MT ferrule to provide a fiber to fiber pitch of $250 \mu \mathrm{m}$. Three fibers were illuminated with white light from a tungsten lamp. A lens was used to collimate the incident light from the inputs and focus the diffracted light from the grating. Based on the fiber pitch and spectral channel spacing a linear dispersion of $\Delta \mathrm{x} / \Delta \lambda=250 / 35=7.143 \mu \mathrm{m} / \mathrm{nm}$ was required in the focal plane of the lens. The linear dispersion of a lens and grating combination used in the Littrow configuration is given by: $\Delta x / \Delta \lambda=2 f \tan (\theta) / \lambda$ Where $f$ is the focal length of the lens, and $\theta$ is the blaze angle of the grating. This equation is valid for wavelengths near the blaze wavelength. The diffraction grating used in this demonstration had a groove density of $400 \mathrm{lines} / \mathrm{mm}$, blaze angle of 9.962 degrees (blaze wavelength $=845 \mathrm{~nm}$ for Littrow mounting), and was gold coated for high reflectivity. Based on the grating parameters, a lens with a focal length of $16 \mathrm{~mm}$ was used to expand and focus the light to and from the fibers. By matching the linear dispersion of the lens and grating combination to the fiber pitch and spectral channel spacing, adjacent spectral channels from a single input are focused to adjacent output fibers. For example, input $A$, sends $\lambda=830,865$, and $900 \mathrm{~nm}$ to outputs 1,2 , and 3 respectively. Furthermore, by spacing the input fibers with the same pitch as the outputs, adjacent inputs send adjacent spectral channels to the same output. Thus, output 3 receives $\lambda=$ 900,865 , and $830 \mathrm{~nm}$ from inputs $\mathrm{A}, \mathrm{B}$, and C respectively. For this device, in general, $\mathrm{N}$ inputs produce $2 \mathrm{~N}-1$ outputs, one of which has all $\mathrm{N}$ wavelengths properly routed and the rest of the outputs forming $\mathrm{N}-1$ pairs of complementary beams. For

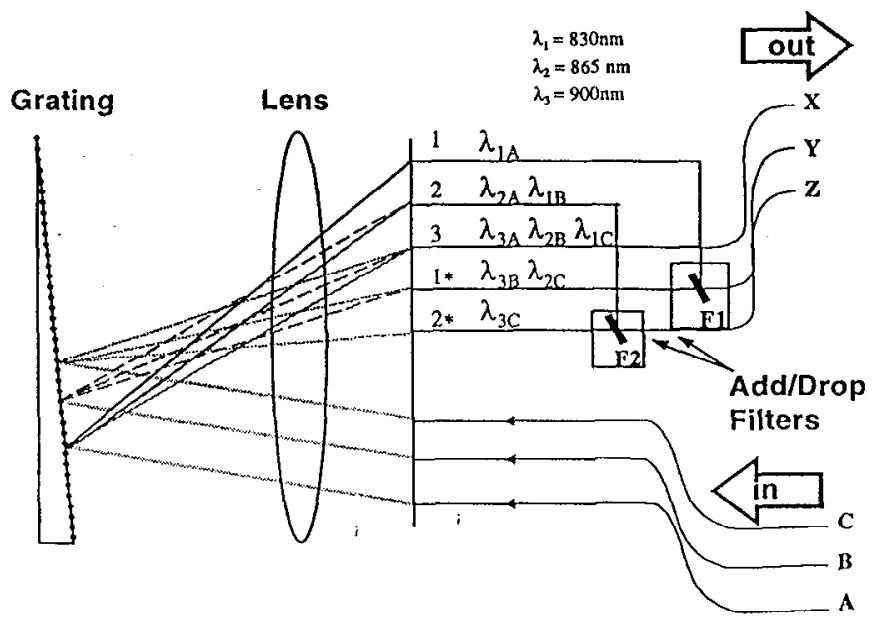

Figure 1. Coarse WDM Router Configuration 
example, output 1 only receives $\lambda=830 \mathrm{~nm}$, while output $1 *$ receives $\lambda=865$ and $900 \mathrm{~nm}$. By combining these pairs of complementary beams, the full routing function is accomplished. Although $2 \times 1$ couplers could be used to combine the beam pairs, it is well known that this type of beam combining incurs a $3 \mathrm{~dB}$ penalty [2]. In order to circumvent this penalty, we used two add/drop filters with different passbands to re-combine the two pairs of complementary output beams. In this application, two inputs are multiplexed onto a single output, one input being reflected by the filter and the other being transmitted through the filter.

\section{Experimental Results}

Figure 2 shows the output spectra from the three outputs (labeled X, Y, and Z in fig. 1), after beam recombination. Each output channel contains all three spectral channels. Although not apparent from this figure, each spectral channel in each output spectrum originates from a different input fiber. In this initial demonstration the channels had a FWHM of $6 \mathrm{~nm}$. The average insertion loss of the device was 8.7 $\mathrm{dB}$ with a standard deviation of 0.81 . Optimizing all of the system components will significantly reduce the insertion loss. For example, the add/drop filters were originally developed for another application and exhibit uncharacteristically high insertion losses, upto a maximum of $4.2 \mathrm{db}$, for the spectral channels

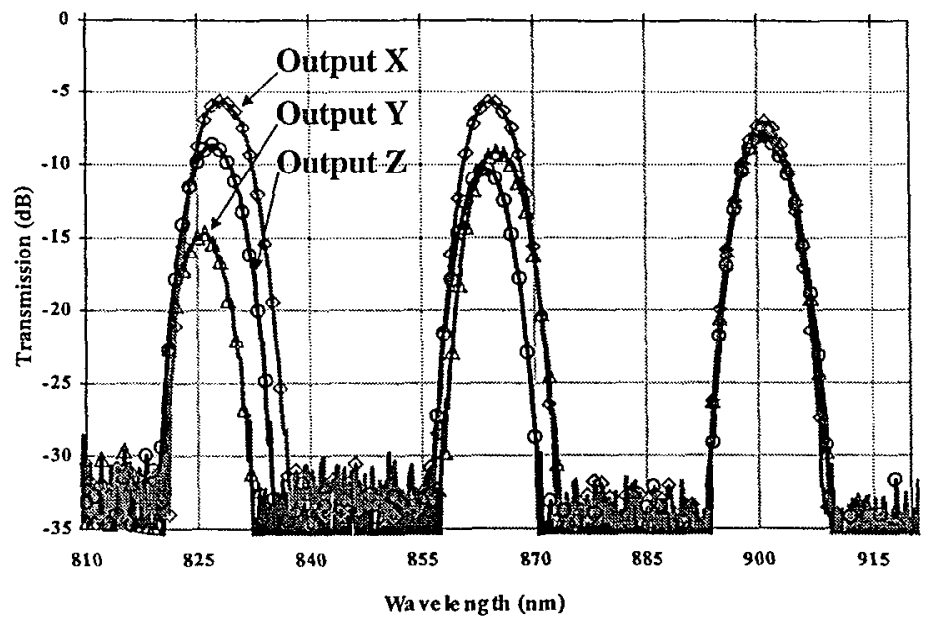
of this experiment. These type of filters are reported to exhibit average insertion losses of 1 to $1.5 \mathrm{~dB}$ when optimized for the wavelengths of interest [3]. Furthermore, the maximum grating efficiency was $87 \%$ ( $0.6 \mathrm{~dB}$ loss). Finally, the maximum insertion loss of $14.7 \mathrm{~dB}$ corresponds to the spectral channel that subtends the largest angle from input to output, travelling from input $A$ to output 1 on figure 1 . This light path experiences very high loss due to vignetting as confirmed by ray tracing. By replacing the lens in the system with a larger diameter $37.5 \mathrm{~mm} f / 1.1$ camera lens the problem of vignetting was eliminated, however, the increased focal length resulted in a linear dispersion of $20.8 \mu \mathrm{m} / \mathrm{nm}$. The new lens supported spectral channel spacings of $12 \mathrm{~nm}$ rather than the $37 \mathrm{~nm}$ required by the available add/drop filters. Although a full system demonstration was not possible, the average insertion loss of the lens and grating combination was reduced to $5 \mathrm{~dB}$. Ulimately, we believe that a device exhibiting insertion losses of 3 to $4 \mathrm{~dB}$ per channel will be attainable.

\section{Summary}

A MMF compatible coarse WDM wavelength router was demonstrated using a diffraction grating and broad-band add/drop filters to achieve wavelength re-use. The scalability of this device to higher channel counts is ultimately limited by the requirements on the lens, the fact that $\mathrm{N}-1$ filter modules are needed to fully route $\mathrm{N}$ wavelengths, and the spatial fill factor of the input/output fiber arrays. The device provides a new tool for WDM over MMF. Furthermore, this design enables a bit-parallel wavelength router by using multiple sets of inputs and outputs. Coupled with multi-wavelength transmitter and receiver arrays such a device will provide high throughput, low latency optical interconnects suitable for massively parallel processing applications.

\section{Acknowledgements}

We thank P. Stefan, C. Bennett, and J. Walker for technical assistance. This work was performed under the auspices of the US Department of Energy by Lawrence Livermore National Laboratory under contract No. W-7405-Eng-48.

\section{References}

1. Takahashi, H. et. al. , "Transmission Characteristics of Arrayed Waveguide NxN Wavelength Multiplexer", J. Lightwave. Tech. V 13 No. 3, 352-360, 1995.

2. Jackel, J. and Tomlinson, W., "An integrated Optical Illusion The lossless single-mode waveguide combiner", Optics \& Photonics News, October , $47-49,1995$.

3. Deri, R. J. et. al. "Simple Fabrication of WDM filters for Byte-Wide Multi-mode Cable interconnects', Proc. 1998 Integrated Photonics Research, 419, 1998. 